\title{
Los riesgos ergonómicos en los trabajadores del Hospital Básico Baños.
}

Ergonomic risks among workers in the Hasic Hospital Bathrooms.

María Fernanda Vargas Ramos ${ }^{1}$, Mariela Ubilluz Garcés. ${ }^{2}$, Geovanny Vega Pérez. ${ }^{3}$, Paúl Fiallos Bucaram. ${ }^{4}$ \& Corina Núñez Hernández. ${ }^{5}$

Recibido: 16-09-2017 / Revisado: 08-11-2017 Aceptado: 10-12-2017/ Publicado: 01-01-2018

\begin{abstract}
.
DOI: $\underline{\text { https://doi.org/10.33262/cienciadigital.v2i1.9 }}$

The objective of the present investigation was to evaluate the ergonomic risks of the workers of the Basic Hospital Baños-Distrito 18D03 who carry out their work activities in the $24 / 7$ system, so that a study was carried out on a population of 137 workers, men and women. Women in the age range between 18 and 60 years That work in the different areas of the institution, for which the field modality was applied, the method used was the validated OWAS test, whose method of application is observational, which evaluates the physical load produced by the postures adopted by the employees Of that institution during the performance of their work duties, in addition, it allows the generation of detailed reports on the frequency with which the worker incurs mistakes about the posture adopted during his work period causing musculoskeletal disorders (TME), which may be affected by the lack of knowledge of the techniques to be used in The relationship man and their work instruments also allows to determine the pace of work and work environment as fundamental aspects of ergonomics, at the same time discloses the factors that affect the health of workers,
\end{abstract}

${ }^{1}$ Universidad Técnica de Ambato, F.C. Humanas. Educación, Ambato, Ecuador, mf.vargas@uta.edu.ec

${ }^{2}$ Universidad Técnica de Ambato, F.C. Humanas. Educación Ambato, Ecuador, mubilluz4235@uta.edu.ec

${ }^{3}$ Universidad Técnica de Ambato, F.C. Humanas. Educación Ambato, Ecuador,jg.vega@uta.edu.ec

${ }^{4}$ Universidad Técnica de Ambato, F.C. Humanas. Educación Ambato, Ecuador, sp.fiallos@uta.edu.ec

${ }^{5}$ Universidad Técnica de Ambato, F.C. Humanas. Educación Ambato, Ecuador, ce.nunez@uta.edu.ec 
we must also add to the labor discomforts of the infrastructure, both In the aspect of the physical space and of mobility of its personnel during the performance of its functions.

Keywords: Musculoskeletal Disorders, Postural Load, Occupational Diseases, Ergonomic Risks, Quality of Life.

\section{Resumen.}

La presente investigación tuvo como objetivo evaluar los riesgos ergonómicos de los trabajadores del Hospital Básico Baños-Distrito 18D03 que desarrollan sus actividades laborales en el sistema 24/7, por lo que se procedió a realizar un estudio a una población de 137 trabajadores, hombres y mujeres, en un rango de edad entre 18 y 60 años que laboran en las diferentes áreas de la institución, para lo que se aplicó la modalidad de campo, el método utilizado fue el test validado OWAS, cuyo método de aplicación es observacional, mismo que valora la carga física producida por las posturas adoptadas por los empleados de dicha institución durante el desarrollo de sus funciones laborales, además permite generar informes detallados sobre la frecuencia con la que el trabajador incurre en los errores sobre la postura adoptada durante su período de trabajo originando trastornos musculoesqueléticos (TME), que tal vez se vea afectada por el desconocimiento de las técnicas que deben ser utilizadas en la relación hombre e instrumentos de trabajo, así mismo permite determinar el ritmo de trabajo y ambiente laboral como aspectos fundamentales de la ergonomía, al mismo tiempo da a conocer los factores que afectan a la salud de los trabajadores, a todo esto debemos sumarle también las incomodidades laborales que presenta la infraestructura, tanto en el aspecto del espacio físico como de movilidad de su personal durante el desempeño de sus funciones.

Palabras Claves: Trastornos Musculo Esqueléticos, Carga Postural, Enfermedades Profesionales, Riesgos Ergonómicos, Calidad de Vida. 


\section{Introducción.}

En la mayoría de instituciones públicas y privadas del país únicamente fijan su objetivo en el rendimiento laboral de sus trabajadores, mientras más réditos, sean económicos o de cualquier tipo, obtengan sus empresas seguirán floreciendo dentro del campo laboral, sin embargo, existen empresas en las que a sus empleados se les capacita en aspectos netamente científicos y de relaciones humanas tal como citan Almirall \& Marroquín (2016) "la mayor necesidad se ve reflejada en la capacitación que precisan nuestros profesionales y técnicos", nunca han pensado en una capacitación ergonómica para precautelar el bienestar de sus colaboradores durante el desempeño de sus tareas diarias dentro de la empresa.

La ergonomía no es más que un método destinado a conocer las diferentes posturas adoptadas por el trabajador durante el desempeño de sus tareas laborales, se lo hace mediante la observación, esta postura hace referencia cuando se aplica a un trabajo estático donde se realiza mediante la aplicación de una parte de cuerpo humano, como por ejemplo la aplicación únicamente de las manos, cuando estamos frente a una pantalla de ecografías, de computadoras y otros, ya que este tipo de trabajo produce una contracción prolongada del músculo provocando malestares tales como dolores, hormigueos, temblores musculares y otros más que afectan grandemente en el desempeño laboral del trabajador, como referencian (Montalvo, Cortés, \& Rojas, 2015).

(Callizo, 2015) describe la ergonomía como "Técnicas preventivas orientadas a abordar los factores de riesgos derivados, principalmente, de la carga de trabajo y de la organización misma".

Es oportuno señalar que el lapso trascurrido entre la exposición del trabajador al riesgo y la presencia de la enfermedad profesional, se lo conoce como periodo de Latencia. Estas patologías se mantienen presentes desde el inicio de cada actividad y con el transcurso del tiempo es preciso valorarlas con la seriedad pertinente, debido a que sus efectos no son evidentes a corto plazo, son progresivos y son causadas por el ejercicio del trabajo mismo, (Agila-Palacios, Colunga-Rodríguez, González-Muñoz, \& Delgado-García, 2016). 
De acuerdo a Cavas (2016) es considerada Enfermedad profesional aquella que "es contraída a consecuencia del trabajo por cuenta ajena o por cuenta propia en las actividades realizadas".

Justamente esta investigación está dirigida a conocer en tiempo real la problemática que afronta el personal de las entidades públicas de salud sobre las precauciones ergonómicas que deben tomar los empleados durante el desempeño laboral, es así que para ello se realizó una investigación en el Hospital Básico Baños de la Provincia de Tungurahua y en los subcentros de salud ubicados en Río Verde, Río Negro, Río Blanco, correspondientes al Cantón Baños, cuya población de estudio es de 137 empleados que realizan diferentes actividades, con horarios de trabajo rotativos, a quienes se procedió evaluar los riesgos ergonómicos que afectan su salud.

Cuando se habla de riesgos ergonómicos en el ámbito de la salud, se hace referencia a las condiciones laborales y las actividades que deben desarrollarse con los cuidados adecuados para evitar la presencia de patologías que afectan y contribuyen en el deterioro de la salud, así como en el desempeño laboral de su personal. En cuanto a las condiciones físicas que ofrece el establecimiento a los trabajadores son cruciales para el buen desempeño laboral, esto se ve reflejado en el bienestar tanto físico como emocional Los trabajadores del Hospital Básico Baños, están expuestos a diversos factores de riesgos, en especial a los ergonómicos, seguramente por el desconocimiento de las técnicas que deben aplicar durante su trabajo, esto ha ocasionado que su salud sea afectada mediante un deterioro temporal o permanente en los trabajadores, lo que incide en el logro de metas y objetivos organizacionales y personales, (Agila-Palacios, Colunga-Rodríguez, González-Muñoz, \& Delgado-García, 2016).

La investigación permitió detectar el factor de riesgos ergonómicos, requiriendo la gestión de la empresa para implementar medidas de prevención, diagnóstico temprano y mitigación de estos con la finalidad de cumplir con el objetivo propuesto en la investigación.

Al citar los riesgos ergonómicos se involucra todos aquellos agentes o situaciones que tienen que ver con la adecuación del trabajo o sus elementos a la fisonomía humana. Representan 
factor de riesgo los objetos, puestos de trabajo, máquinas, equipos y herramientas cuyo peso, tamaño, forma y diseño pueden provocar sobre-esfuerzo, así como posturas y movimientos inadecuados que traen como consecuencia fatiga física y lesiones osteomusculares, siendo el caso en el sector hospitalario y en especial en el área de emergencias la utilización de equipos hospitalarios que permiten la movilidad de los pacientes, los mismos que no siempre son manipulados con las medidas de seguridad requeridas. Se puede evidenciar también que el personal administrativo del hospital registra afecciones derivadas de los riesgos ergonómicos, lo que se puede corroborar mediante las diversas investigaciones realizadas en el sector hospitalario, (Oñate, Chica, Jaraba, \& Jaraba, 2015).

En relación a los trastornos musculoesqueléticos según Gómez-Ceballos (2016), se consideran una importante causa de ausentismo laboral, pueden empobrecer a los trabajadores y a sus familias, reducir la productividad y la capacidad de trabajo, y aumentar drásticamente los gastos de la atención en salud. Además, Bettiol, 2016 (como se citó en Sbriller, 2016) comenta que se excluye a personas que por la gravedad de las secuelas no podrán continuar con su trayectoria laboral.

Caicedo, Manzano, Gómez, \& Gómez (2015). En su investigación se afirma que: se encontró que las medidas de control y prevención están relacionadas y enfocadas desde la adaptación de dispositivos que previenen la aparición del dolor lumbar, hasta medidas administrativas como el levantamiento de cargas divididas, la disminución de la carga, la implementación de tiempo de recuperación y de la carga máxima aceptable para los trabajadores.

Soriano Tarín, Rodríguez-Caro, Pascual Sagastagoiti, \& Del Campo Balsa (2016). Expresa que la valoración de la aptitud o capacidad para trabajar es importante, con el objetivo de verificar si existe una adaptación o un desequilibrio entre las exigencias psicofísicas del puesto y la capacidad funcional del trabajador.

De acuerdo con Aliaga, Villarroel, \& Cossio, (2016) los factores principales de riesgos que afectan a la población en estudio, aportando a la creación de estrategias que busca la 
prevención ergonómica, indicando dónde es adecuado destinar recursos para lograr intervenciones eficaces.

Souza de Souza, Cortez Antunes, Gomes, T., \& Santana Ferreira, (2016) afirma que cuando las condiciones de trabajo sobrepasan los límites tolerables del organismo, la probabilidad de provocar una enfermedad de índole laboral TME es significativa, es necesario realizar una revisión íntegra en busca de los factores causantes de estos problemas y poder ofrecer actividades educativas para minimizar la exposición de enfermedades profesionales.

En la investigación de Galvis, Pérez, Ramírez, Batancur, \& Gómez, (2015) se observa que en la empresa Solomoflex, los trabajadores mantienen posturas incorrectas y movimientos forzados, lo que afecta a los segmentos corporales, como miembros superiores y espalda.

Chaves, Martínez, \& López, (2014). Chávez en su investigación sobre la carga física postural y su relación con sus trastornos musculoesquelético concluye que: para el correcto desempeño laboral en el área de despacho de la empresa a objeto de estudio, es importante que el departamento de seguridad y salud, implemente un programa de vigilancia epidemiológica para trastornos musculoesqueléticos dentro de las actividades de promoción y prevención de la empresa.

Angulo \& Ramírez, (2016). En su estudio "Relación entre la calidad de vida en salud y la carga física en cuidadores de personas con enfermedad de Alzheimer" afirman que el acumulo de la sintomatología y el deterioro de la calidad de vida en la salud de los cuidadores, pueden llevar a un aumento en la consulta a los servicios de salud debido a las dolencias generadas por la fatiga muscular a la que se encuentran expuestos, las personas cuidadoras poseen un firme compromiso para sus pacientes, lo cual no les permite dedicar tiempo para si mismo.

Pinto, (2015). La investigación destaca que: el grupo ergo manifiesta satisfacción de haber conocido la disciplina de la ergonomía, poder aplicarla en su labor diaria, proponer medidas de control y luego verlas implementadas, así también, concluye que un programa de 
ergonomía participativa puede ser una buena relación costo-beneficio para las proyecciones de la empresa.

Estos autores concuerdan en que las enfermedades profesionales, son ocasionadas directamente por el ejercicio de la profesión que ejecuta una persona y que pueden originar incapacidad, en casos extremos la muerte del trabajador, por lo tanto, para registrarla como tal se debe analizar e indagar la manera en que fue adoptada, es decir, que no se confunda entre enfermedad profesional y accidente laboral, puesto que la enfermedad profesional se suscita con el paso del tiempo y el accidente laboral es prácticamente fortuito e impredecible y guarda relación con la ejecución de su trabajo.

Zamora, Montesdeoca, Negrin, \& Párraga (2016), informan que en su investigación se observó que en la mayoría de los casos el trabajador tiene una postura incorrecta al momento de realizar las actividades como: estar de pie y en frecuente movimiento, de pie con rodillas ligeramente flexionadas, sentado sin levantarse con frecuencia, agachado con la espalda doblada, entre otras, los cuales manifiestan que realizan sus tareas en posturas no adecuadas ocasionando molestias frecuentes, debido a la actividad requiere constantemente esfuerzos físicos, el ambiente de trabajo también influye en el desenvolvimiento de la tarea por lo cual a veces realizan posturas dolorosas y fatigantes, levanta o mueve carga pesada, mantiene una misma postura, movimientos repetitivos de manos, ruido entre otros aspectos que afectan la seguridad y salud a un largo plazo. Por lo tanto, Universidad de la salud, Kamei, Takahashi, Omori, Arimori, \& Hishinuma, "et al.”., (2016), argumentan que: es necesario promover no sólo la prevención de enfermedades, sino también los servicios de salud específicos para los residentes en la comunidad local.

Este trabajo busca hacer conciencia sobre el objeto de aprendizaje, se convierte en la primera condición necesaria para el proceso formativo en busca de una correcta atención. Según Rincones \& Castro, (2016). Indican que se estableció que la interrelación entre las condiciones de salud de los trabajadores y la vulnerabilidad ante la exposición de los riesgos laborales requieren estrategias beneficiosas entre las actividades de protección, prevención 
de riesgos y promoción de la salud en el trabajo, cuyo resultado final se traduciría en un trabajo saludable.

Rincones (2016) en su investigación "El estudio científico de las relaciones del hombre y del medio de trabajo" menciona que la aplicación conjunta de algunas ciencias biológicas y ciencias de la ingeniería, para asegurar entre el hombre y el trabajador una óptima adaptación mutua con el fin de incrementar el rendimiento del trabajador y contribuir a su propio bienestar.

En general, estos autores concuerdan que la ergonomía está enfocada en el estudio del trabajo: en su espacio físico, ambiente, diferentes tipos de desgaste y todo aquello que pueda poner en peligro la salud del trabajador; más que en la productividad, busca medidas que se puedan aplicar para la prevención de accidentes y centrarse en la modificación de las condiciones de trabajo, en pro del bienestar del trabajador.

Bravo \& Espinoza, (2016) destacan en su investigación que la incorporación de la ergonomía en el ámbito laboral ha propuesto mejoras al respecto tanto en el bienestar de los trabajadores como en la productividad en las empresas; sin embargo, es necesario que exista eficiencia y seguridad en los trabajos, debido a que es importante que el personal cuente con condiciones favorables para desarrollar sus actividades.

Las escasa prevención y capacitación acerca de las enfermedades profesionales conllevan a que sean diagnosticadas en forma tardía, lo cual afecta la calidad de vida del trabajador y sus familias.

Souza et al. (2016) expresa que es preciso la revisión integra en busca de las enfermedades profesionales para poder describir los factores causantes de estos problemas y poder ofrecer actividades educativas para minimizar la exposición de enfermedades profesionales.

Cabe mencionar que, según la legislación de España, son consideradas enfermedades profesionales únicamente las que se encuentran en el cuadro aprobado por el RD 1299/2006, 
las cuales serán provocadas de acuerdo a los agentes que se encuentran en dicho cuadro. Mientras que, en la República del Ecuador, esta competencia le pertenece al Instituto Ecuatoriano de Seguridad Social IESS, a través del Seguro General de Riesgos del Trabajo quienes emitieron el Reglamento del Seguro General de Riesgos del Trabajo (Resolución CD $513)$.

Serra, (2016), este trabajo busca indagar las causas más frecuentes de discapacidad o enfermedades crónicas producidas por la necesidad de mantener posturas forzadas limitadas al lugar de trabajo, produciendo dolencias de índole osteomuscular.

Es considerada una manifestación patológica en el organismo del individuo, contraída en el ejercicio de sus ocupaciones laborales, y que estén estimuladas por la gestión o sustancias que se indican para la descrita Enfermedad Profesional, la cual puede dejar una incapacidad valorativa.

Pereira, Da Silva, \& Dominguez, (2016) afirman que hay factores que contribuyen para el lumbago y entre "los individuales se encuentran: edad, sexo, índice de masa corpórea, desequilibrio muscular, fuerza muscular, condiciones socioeconómicas, presencia de otras enfermedades y los factores laborales", este trabajo se encarga de conocerlos.

El personal de emergencias y de enfermería no son los únicos que están expuestos a trastornos que afectan su salud, siendo el personal médico también parte de este riesgo como lo menciona Moreno, (2016) en su estudio "Ergonomía En La Práctica Odontológica”, afirma que los trastornos músculo esqueléticos incluyen todas aquellas alteraciones que recaen sobre la columna vertebral y/o los miembros superiores o inferiores, afectando estructuras musculoesqueléticas y se consideran una patología típica de la profesión, mediante la Ergonomía, se pretende mejorar la interacción del clínico como ser humano, con su equipo o maquinaria de trabajo para promover su salud y seguridad, evitando así lesiones o daños causados por posturas inadecuadas durante su desempeño. 
Así también Escudero, Aprili, Muñoz, De La Cruz, \& Moscoso (2016), concluyen que el síndrome del Túnel Carpiano de origen laboral es frecuente en odontólogos y está relacionado con esfuerzos manuales intensos, posiciones manuales forzadas, movimientos repetitivos, herramientas vibratorias y presión en muñeca-mano.

Así lo argumenta Hodelín, De los Reyes, Hurtado, \& Batista, (2016), evidencian que los movimientos repetidos durante un periodo largo de tiempo, puede llevar a la compresión del mediano, uno de los nervios principales que pasa por la muñeca y la mano. El resultado es una sensación de debilidad, hormigueo, entumecimiento y quemadura seguido de un dolor agudo. El desorden se conoce como síndrome del túnel carpiano y se puede causar por trabajar constantemente en los teclados y con los mouses de las computadoras. El equipo ergonómicamente diseñado incluye soportes para las muñecas, bandejas para los teclados y pelotas de rastreo para evitar la presión en los nervios. Otras medidas de prevención que se pueden sugerir son los ejercicios regulares con la muñeca y descansos cortos. El síndrome del túnel carpiano se trata con compresas calientes y frías, tablillas y antiinflamatorios. En casos severos los procedimientos quirúrgicos se aplican para remover o mover el ligamento que está presionando el nervio y causando el dolor.

Profesores de la Universidad de Santiago de Chile. Malchaire Jacques., (2016) "Sobane: Una Estrategia De Gestion Del Riesgo A Considerar." concluye que, para la reducción de factores de riesgo y situaciones de trabajo, se debe considerar la implementación de un programa de fortalecimiento de la seguridad y salud ocupacional.

Finalmente, Ordoñez, Gómez, \& Calvo, (2016) en su estudio manifiestan que estos diagnósticos se relacionaron con planificación ineficaz de actividades, deterioro de la interacción social, afrontamiento ineficaz de la comunidad, conflicto de decisiones y riesgo de contaminación.

\section{Materiales y Métodos.}

La presente investigación se la realizó en el Hospital de la ciudad de Baños provincia de Tungurahua, perteneciente al Distrito 18D03. La población se conformó con 137 personas 
equivalente al cien por ciento del universo y se utilizó el software "Ergonautas" con el método de evaluación Ergonómica OWAS (Ovako Working Analysis System) que cuenta con un test avalado por la Universidad Pontificia de Valencia - España. Debido a que se requirió evaluar la carga postural que mantienen las principales posturas de espalda, miembros superiores y miembros inferiores en las áreas y durante el desarrollo del trabajo; parte desde la observación de las diferentes posturas en intervalos regulares, se estableció un periodo de análisis necesario para el registro de posturas adoptadas por el trabajador. La frecuencia de observación dependió de la frecuencia con la que el trabajador cambió de postura y de la variedad, se observó la tarea durante el periodo definido y se registraron las posturas a través de fotografías para su posterior análisis, finalmente se realizaron los cálculos expuestos en apartados posteriores.

En la Tabla No 1 se detalla la población por departamentos que fueron objetos de estudio en el periodo temporal correspondiente al primer semestre del año 2016, para lo cual se relacionaron los riesgos ergonómicos presentes en los puestos de trabajo con las enfermedades profesionales, por ende, los datos fueron levantados acorde a los requerimientos del método.

Tabla No. 1 Población

\begin{tabular}{lcccc}
\hline \multirow{2}{*}{\multicolumn{1}{c}{ Objeto de estudio }} & \multicolumn{2}{c}{ Frecuencia } & \multirow{2}{*}{ Total } & Porcentaje \\
\cline { 2 - 4 } & Masculino & Femenino & & \\
\hline Administrativo & 0 & 2 & 2 & $2 \%$ \\
Atencion integral en salud & 23 & 49 & 72 & $53 \%$ \\
Direccion distrital de salud & 2 & 0 & 2 & $2 \%$ \\
Farmacia & 0 & 3 & 3 & $2 \%$ \\
Gestion distrital de tecnologias de la & 2 & 0 & 2 & $1 \%$ \\
informacion y comunicación & 19 & 6 & 25 & $18 \%$ \\
Servicios generals & 2 & 0 & 2 & $1 \%$ \\
Talento humano & 8 & 11 & 19 & $14 \%$ \\
Unidad distrital de provision y calidad de los & 8 & 2 & 10 & $7 \%$ \\
servicios de salud & $\mathbf{6 4}$ & $\mathbf{7 3}$ & $\mathbf{1 3 7}$ & $\mathbf{1 0 0 \%}$ \\
Ecu 911 & & & & \\
Total & & & & \\
\hline
\end{tabular}

Fuente: Hospital Básico Baños

Antes de aplicar el método se realizó un análisis de todas las actividades desarrolladas por el trabajador a lo largo de su jornada laboral con la finalidad de identificar si las fases de trabajo 
son homogéneas o heterogéneas, ya que de esto depende si la evaluación es simple o multifase, de tal manera que si se han establecido varias fases la evaluación se realizará separadamente para cada una de ellas. Por otra parte, también se determina el tiempo y frecuencia de observación, número representativo de muestras realizadas, el medio de registro de las posturas y movimientos adoptados por un periodo de 40 minutos de observación.

Posteriormente se adquiere la licencia de "Ergonautas" para la utilización del software del Método OWAS. Definidas las fases, el periodo de observación y la frecuencia de muestreo; se registran las posturas de espalda a través del código ( 1 erguida, 2 inclinada adelante o atrás, 3 girada o inclinada hacia un lado, 4 girada o inclinada adelante y hacia un lado), brazos con los códigos ( 1 ambos brazos por debajo de los hombros, 2 un brazo al nivel o por encima del hombro, 3 ambos brazos al nivel o por encima de los hombros), piernas con los códigos ( 1 sentado, 2 de pie con las piernas rectas, 3 de pie cargando el peso en una pierna recta, 4 de pie o agachado con las piernas dobladas, 5 de pie o agachado con una rodilla doblada, 6 arrodillado sobre una o ambas rodillas, 7 andando o en movimiento) y carga/fuerza el peso o fuerza es menor que $10 \mathrm{Kg}$, el peso o fuerza es mayor a $10 \mathrm{Kg}$ menor a $20 \mathrm{Kg}$, 3 el peso o fuerza es mayor a $20 \mathrm{Kg}$.), las mismas que se registran a través de medios digitales para posteriormente ser analizados; finalmente se ingresan los datos recabados en el software, dónde se realizan los cálculos necesarios para obtener la valoración del riesgo ergonómico debido a la adopción de posturas en el desarrollo de la tarea, procediendo a categorizarlo en dos distintos niveles de análisis (Básico y Avanzado). La evaluación de los riesgos se la realiza en función de los valores alcanzados en cada parte del cuerpo y por supuesto de trabajo, para luego clasificarlos en función de su grado de afectación, lo que se resume en la Tabla No 2.

Tabla No. 2 Gráfico de los grados de afectación

\begin{tabular}{|c|c|}
\hline Riesgo & Información \\
\hline 1 & Postura normal y natural sin efectos dañinos sobre el sistema músculo-esquelético. \\
\hline 2 & Postura con posibilidad de causar daño al sistema músculo-esquelético. \\
\hline 3 & Postura con efectos dañinos sobre el sistema músculo-esquelético. \\
\hline 4 & $\begin{array}{l}\text { La carga causada por esta postura tiene efectos sumamente dañinos sobre el sistema } \\
\text { músculo-esquelético. }\end{array}$ \\
\hline
\end{tabular}

Fuente: Método OWAS - Ergonautas

En el nivel de análisis básico la identificación inicial de riesgos permite la detección de factores de riesgo en los puestos de trabajo de manera directa. Por otra parte, en caso de que 
los riesgos ya han sido detectados o muestran evidencia de su presencia, se procede con el nivel de análisis avanzado que se enfoca en el trabajador, a fin de evaluar el factor de riesgo asociado entre las fases que lo componen, por ejemplo: además de las posturas adoptadas debe evaluarse la repetitividad de movimientos y la carga física presente en los mismos.

De tal manera que, en caso de contar con el análisis estadístico de los registros médicos laborales del personal son de gran ayuda para esta detección inicial de riesgos, otra manera de evidenciar con buenos indicadores de la presencia de riesgos son, por ejemplo: la presencia de lesiones agudas (lumbalgias, fatiga física, hernias discales, ciáticas), lesiones crónicas que pueden ser consideradas enfermedades profesionales (epicondilitis, síndrome del túnel carpiano, tendinitis mano - muñeca, síndrome de vibración mano - brazo).

Por lo cual, se evaluó el principal factor de riesgo que da origen a los Trastornos MúsculoEsqueléticos (TME) en la zona cuello-hombros, para así considerar si es necesaria la intervención desde el punto de vista ergonómico. Lo anteriormente mencionado se encuentra acorde y en benefício del Comité Interno de Seguridad y Calidad de Vida del Hospital Básico Baños, mismo que se conforma con los delegados de los diferentes Departamentos y son los encargados de velar por la salud y condiciones de vida del personal.

\section{Resultados y Discusión.}

Una vez ingresados en el Software los datos obtenidos en la investigación, se evidencia el nivel de riesgo al que se encuentra expuesto el personal mediante la metodología de evaluación OWAS, de tal manera que es posible presentar en la Tabla No 3 las posturas que mayor daño producen en cada puesto evaluado.

Tabla No. 3 Posturas que mayor daño causan.

No. Puesto Evaluado

Postura más crítica 
ISSN: 2602-8085

Vol. 2, $\mathrm{N}^{\circ} 1$, p. 8-18, enero - marzo, 2018

\begin{tabular}{|c|c|c|c|c|c|c|}
\hline & & $\begin{array}{c}\text { Tipo de } \\
\text { Evaluación }\end{array}$ & Código & Postura & $\begin{array}{c}\text { Nivel } \\
\text { Riesgo }\end{array}$ & Frecuencia \\
\hline 1 & Conductor del sector público & Simple & 1 & Piernas-Sentado & 1 & $100 \%$ \\
\hline 2 & Auxiliar de farmacia & Simple & 4 & $\begin{array}{l}\text { Espalda doblada } \\
\text { con giro }\end{array}$ & 4 & $33.33 \%$ \\
\hline 3 & Auxiliar administrativo & Avanzado & 4 & $\begin{array}{l}\text { Espalda doblada } \\
\text { con giro }\end{array}$ & 4 & $25 \%$ \\
\hline 4 & $\begin{array}{l}\text { Técnico médico de } \\
\text { rehabilitación y terapia física }\end{array}$ & Avanzado & 4 & $\begin{array}{l}\text { Espalda doblada } \\
\text { con giro }\end{array}$ & 4 & $25 \%$ \\
\hline 5 & $\begin{array}{l}\text { Asistente administrativa - } \\
\text { secretaria }\end{array}$ & Avanzado & 2 & Espalda doblada & 2 & $60 \%$ \\
\hline 6 & Director distrital & Simple & 2 & $\begin{array}{l}\text { Espalda doblada } \\
\text { con giro }\end{array}$ & 2 & $33.33 \%$ \\
\hline 7 & Auxiliar administrativo & Avanzado & 4 & $\begin{array}{l}\text { Espalda doblada } \\
\text { con giro }\end{array}$ & 4 & $66.67 \%$ \\
\hline
\end{tabular}

Fuente: Hospital Básico Baños - Método OWAS.

De igual manera, se extraen los porcentajes de afección de los niveles de riesgo correspondientes a la parte del cuerpo evaluada en cada puesto de trabajo seleccionado tal como se puede apreciar en la Tabla No 4, con lo cual se discierne la parte del cuerpo que mayor exposición al deterioro músculo esquelético presenta en la consecución de sus actividades de trabajo.

Tabla No. 4 Grados de afectación.

No.

Puesto Evaluado

Niveles de Riesgo Evaluado 
Vol. 2, $\mathrm{N}^{\circ} 1$, p. 8-18, enero - marzo, 2018

\begin{tabular}{|c|c|c|c|c|c|c|}
\hline & & $\begin{array}{c}\text { Parte de } \\
\text { Evaluación }\end{array}$ & NR4 & NR3 & NR2 & NR1 \\
\hline \multirow{3}{*}{1} & \multirow{3}{*}{ Conductor del sector público } & Espalda & $33.33 \%$ & $50.00 \%$ & $0.00 \%$ & $16.67 \%$ \\
\hline & & Brazos & $0.00 \%$ & $33.33 \%$ & $66.67 \%$ & $0.00 \%$ \\
\hline & & Piernas & $16.67 \%$ & $33.33 \%$ & $50.00 \%$ & $0.00 \%$ \\
\hline \multirow{3}{*}{2} & \multirow{3}{*}{ Auxiliar de farmacia } & Espalda & $33.33 \%$ & $0.00 \%$ & $70.00 \%$ & $0.00 \%$ \\
\hline & & Brazos & $0.00 \%$ & $90.00 \%$ & $10.00 \%$ & $0.00 \%$ \\
\hline & & Piernas & $16.67 \%$ & $30.00 \%$ & $50.00 \%$ & $20.00 \%$ \\
\hline \multirow{3}{*}{3} & \multirow{3}{*}{ Auxiliar administrativo } & Espalda & $30.33 \%$ & $0.00 \%$ & $16.67 \%$ & $83.33 \%$ \\
\hline & & Brazos & $0.00 \%$ & $0.00 \%$ & $0.00 \%$ & $100.00 \%$ \\
\hline & & Piernas & $0.00 \%$ & $0.00 \%$ & $16.67 \%$ & $83.33 \%$ \\
\hline \multirow{3}{*}{4} & \multirow{3}{*}{$\begin{array}{l}\text { Técnico médico de } \\
\text { rehabilitación y terapia física }\end{array}$} & Espalda & $0.00 \%$ & $0.00 \%$ & $60.00 \%$ & $40.00 \%$ \\
\hline & & Brazos & $0.00 \%$ & $0.00 \%$ & $0.00 \%$ & $100.00 \%$ \\
\hline & & Piernas & $0.00 \%$ & $0.00 \%$ & $40.00 \%$ & 60.00 \\
\hline \multirow{3}{*}{5} & \multirow{3}{*}{$\begin{array}{l}\text { Asistente administrativa - } \\
\text { secretaria }\end{array}$} & Espalda & $0.00 \%$ & $0.00 \%$ & $57.14 \%$ & $42.86 \%$ \\
\hline & & Brazos & $0.00 \%$ & $0.00 \%$ & $28.57 \%$ & $71.43 \%$ \\
\hline & & Piernas & $0.00 \%$ & $0.00 \%$ & $0.00 \%$ & $100.00 \%$ \\
\hline \multirow{3}{*}{6} & \multirow{3}{*}{ Director distrital } & Espalda & $0.00 \%$ & $37.50 \%$ & $0.00 \%$ & $62.50 \%$ \\
\hline & & Brazos & $0.00 \%$ & $75.00 \%$ & $25.00 \%$ & $0.00 \%$ \\
\hline & & Piernas & $0.00 \%$ & $37.50 \%$ & $37.50 \%$ & $25.00 \%$ \\
\hline \multirow{3}{*}{7} & Auxiliar & Espalda & $0.00 \%$ & $50.00 \%$ & $33.33 \%$ & $16.67 \%$ \\
\hline & administrativo & Brazos & $0.00 \%$ & $0 . \%$ & $33.33 \%$ & $66.67 \%$ \\
\hline & & Piernas & $0.00 \%$ & $33.33 \%$ & $16.67 \%$ & $50.00 \%$ \\
\hline
\end{tabular}

Fuente: Hospital Básico Baños - Método OWAS.

Finalmente en la Tabla No 5, se presentan los resultados de mayor a menor en relación al impacto o efecto que generan sobre la Seguridad y Salud de los trabajadores, además, se presentan las acciones correctoras en función del tiempo de holgura que se dispone para implementarlas en cada puesto de trabajo, para lo cual se tomará en cuenta las características de los movimientos, su repetitividad y la carga postural que manejan.

Tabla No. 5 Resultados Test OWAS. 


\begin{tabular}{llcl}
\hline \multicolumn{1}{c}{ Encuestados } & Riesgo & Frecuencia & \multicolumn{1}{c}{ Efectos y Acciones Correctoras } \\
\hline $\begin{array}{l}\text { Auxiliar administrativo } \\
\text { Nutrición }\end{array}$ & 4 & $66,67 \%$ & $\begin{array}{l}\text { La carga causada tiene efectos altamente } \\
\text { dañinos, se requiere tomas acciones } \\
\text { inmediatamente. }\end{array}$ \\
$\begin{array}{llll}\text { La carga causada tiene efectos altamente } \\
\text { dañinos, se requiere tomas acciones }\end{array}$ \\
$\begin{array}{l}\text { inmediatamente. } \\
\text { Auxiliar administrativo farmacia }\end{array}$ & 4 & $33,33 \%$ & $\begin{array}{l}\text { La carga causada tiene efectos altamente } \\
\text { dañinos, Se requiere tomas acciones } \\
\text { inmediatamente. }\end{array}$ \\
$\begin{array}{l}\text { Técnico Médico de } \\
\text { Rehabilitación y Terapia } \\
\text { Física }\end{array}$ & 4 & $25,00 \%$ & $\begin{array}{l}\text { La carga causada tiene efectos altamente } \\
\text { dañinos, se requiere tomas acciones } \\
\text { inmediatamente. }\end{array}$ \\
$\begin{array}{l}\text { Asistente administrativa - } \\
\text { secretaria }\end{array}$ & 2 & $60,00 \%$ & $\begin{array}{l}\text { Postura con posibilidad de causar daños, se } \\
\text { requiere acciones en un futuro cercano. }\end{array}$ \\
$\begin{array}{l}\text { Director Distrital } \\
\text { Conductor del Sector Público }\end{array}$ & 1 & $100,00 \%$ & $\begin{array}{l}\text { Postura normal sin efecto, no requiere } \\
\text { acción. }\end{array}$ \\
\hline
\end{tabular}

Fuente: Hospital Básico Baños - Método OWAS.

A diferencia de lo realizado en la investigación de Ordoñez, Gómez, \& Calvo, (2016) la cual se enfoca en la localización de determinadas lesiones o TME y la relación que estos mantienen con el rango de edad de los trabajadores que las padecen, lo cual sugiere que la presencia de dichas dolencias no es producida únicamente por las condiciones de trabajo, sino también por condiciones individuales siendo factores condicionantes para la presencia de dolor. Lo cual es lógico, pero no completamente cierto para la presente investigación, debido a que los riesgos ergonómicos identificados y evaluados se encuentran directamente relacionados a la carga-postural propia de las actividades que se ejecutan en los puestos de trabajo analizados, lo cual lleva intrínseco un potencial dañino para la salud física del trabajador, debido a que éste no cuenta con la debida formación en lo referente a posturas ergonómicas de trabajo y manejo de cargas, además, se puede apreciar que los trabajadores del sector operativo son precisamente los que evidencian mayor riesgo, siendo así los candidatos idóneos a desarrollar una enfermedad producida por Trastornos Músculo Esqueléticos a pesar de que promedio de edad no supera los treinta y ocho años. 
Es posible discernir que la parte del cuerpo que se encuentra mayormente afectada por los movimientos y esfuerzos ocasionados en la consecución del trabajo se encuentran en la espalda, dentro de la cual, la postura corporal que genera mayor nivel de afección en los diferentes puestos de trabajo es "Espalda Doblada con Giro", misma que por el nivel potencial de riesgo a desarrollado TME entre el personal operativo del Hospital, las principales afecciones son: síndrome cervical por tensión o servicalgia, lumbalgias, hernias discales; lo cual disminuye sustancialmente el rendimiento laboral y la calidad de vida de los trabajadores. Esta situación es completamente diferente de lo que se encuentra en la investigación de Agila (4), quién sugiere que en la aparición de estos síntomas intervienen diversos factores, como por ejemplo: el hábito tabáquico, el nivel de práctica de actividad física, el género, factores genéticos individuales. Se debe destacar que, en la presente investigación, los puestos conformados por varias fases son los más vulnerables, debido a la combinación de movimientos por realizar, tomando en consideración que la carga o peso en traslado/manipulación no se trata de una herramienta u objeto, al contrario son seres humanos que presentan diversas afecciones en su salud y que deben ser trasladados con las debidas precauciones; esto se refleja en los porcentajes de exposición en que se presenta esta situación, que en combinación con la carga postural adoptada por el trabajador, origina mayor grado de severidad en el daño, lo cual se evidencia en las extremidades superiores con la presencia de tendinitis en hombro, epicondilitis lateral o codo de tenista y túnel carpiano a nivel de muñeca, siendo las afecciones identificadas en la investigación.

Se puede notar que el grupo muscular correspondiente a las piernas obtiene niveles de riesgo con efectos dañinos sobre el sistema músculo esquelético, para los cargos de Conductor del Sector Público, Auxiliar de Farmacia, Director Distrital y Auxiliar Administrativo, sin embargo, éstos puestos de trabajo presentan características diferentes en lo referente a repetitividad de movimientos, posturas forzadas, cantidad de trabajo estático/dinámico, etc. Es así que manifiesta Agila-Palacios, et al, (2016) "Los trabajadores de mantenimiento al realizar tareas con levantamientos frecuentes, trasladar objetos livianos o pesados, manejo manual de materiales y al efectuar prolongados turnos de trabajo, pueden estar expuestos a factores de riesgos ergonómicos, añadidos a otros factores tales como los organizacionales, 
individuales y ambientales, pueden ocasionar síntomas musculo-esqueléticos, afectando el estado de salud del trabajador".

En concordancia con lo dispuesto por la Constitución de la República del Ecuador 2008, (2008) en su artículo 326, numeral cinco "Toda persona tendrá derecho a desarrollar sus labores en un ambiente adecuado y propicio, que garantice su salud, integridad, seguridad, higiene y bienestar", de tal manera que como medida de prevención y/o mitigación el orden lógico de reducción de riesgos es, primero en la fuente de riesgo, segundo en el medio de transmisión de dicho riesgo y tercero en el receptor, por lo tanto, esto sugiere, que se revisen las condiciones subestándar de los puestos de trabajo, es decir el entorno y mobiliario con el que cuentan, para así organizar de mejor manera los puestos de trabajo y reducir las acciones subestándar que cometen los trabajadores.

Ciertamente los puestos de trabajo presentan diversas condiciones en su entorno, originando así varios movimientos corporales de tal manera que los grupos musculares que se ven expuestos son diferentes, por lo cual, el nivel de riesgo en cada caso es distinto, los niveles de riesgo que predominan están directamente relacionados con las partes del cuerpo que adoptan cargas posturales extremas y las mantienen por un periodo de tiempo. Investigaciones anteriores como la de Morán, (2016) argumenta que durante las labores es común que se realicen movimientos y/o adopten posturas forzadas que puedan producir lesiones y/o daño, y que permanecen en una postura fija por tiempo prolongado sin realizar una pausa o descanso agravando ergonómicamente la situación.

Debiendo tomar en cuenta los Niveles de Riesgo para proponer las medidas correctivas en cada caso, sin dejar de lado la frecuencia que presentan las mismas. Por lo cual, los puestos de trabajo deben ser analizados por separado, para identificar el nivel de riesgo y la parte del cuerpo que requiere mayor atención. Sin duda alguna, los puestos de trabajo que presentan mayor repercusión en relación a la generación de TME son los del nivel operativo, debido a que sus actividades requieren de mayor cantidad de movimientos con carga postural y física, lo cual ocasiona mayor desgaste. Como así lo mencionan Guizado \& Zamora, (2014) en su estudio que existe una "correlación moderada media positiva" (Rho de Spearman 0.517) entre 
los riesgos ergonómicos (posturas forzadas prolongadas $(0.718)$ y movimientos corporales (0.649) ambas con un nivel de correlación alta positiva) con la lumbalgia ocupacional en la dimensión aguda, y los riesgos peso fuerza (0.619), movimientos corporales (0.603) y posturas forzadas prolongadas $(0.436)$ presentan una "correlación moderada media y alta positiva" respectivamente, tienen un mayor nivel de relación con la lumbalgia sub aguda y la postura corporal (0.055), peso fuerza (0.158) ambas con una "correlación baja positiva", no prestan un mayor nivel de relación con la lumbalgia crónica.

\section{Conclusiones.}

- Se diagnosticó que los riesgos ergonómicos como carga-postural afectan a los colaboradores del Hospital Básico Baños en el transcurso de su vida laboral, algunos sin transcendencia, en otros casos el daño va tomando gravedad e importancia por la presencia de diferentes patologías, con el paso del tiempo dichas patologías llegan a convertirse en enfermedades profesionales que condicionan su calidad de vida.

- Las principales afecciones a la salud del personal que labora en el Hospital Básico Baños, de acuerdo a cada grupo muscular son: a nivel de espalda (servicalgias, lumbalgias y hernias discales), en brazos (tendinitis en hombro, epicondilitis lateral y túnel carpiano), por último, en piernas existen (varices y bursitis en las rodillas).

- La institución brinda a sus trabajadores escasa seguridad y capacitación en términos de condiciones laborales, medidas preventivas, por ende, los colaboradores del distrito se ven afectados por riesgos ergonómicos, desarrollando enfermedades profesionales ocasionadas por TME, en el cumplimiento de sus funciones laborales.

- La inadecuada infraestructura dificulta la distribución del espacio, obstaculizando la movilidad, provocando cargas posturales inadecuadas, de manera que perjudica directamente el desempeño de funciones, teniendo en cuenta que las actividades laborales se las realiza 24/7 lo que provoca una carga de trabajo tanto en el día como en la noche produciendo cansancio físico, mental y emocional, que afecta la productividad de la organización. 


\section{Referencias bibliográficas.}

Agila-Palacios, E., Colunga-Rodríguez, C., González-Muñoz, E., \& Delgado-García, D. (2016). Síntomas Músculo-Esqueléticos en Trabajadores Operativos del Área de Mantenimiento de una Empresa Petrolera Ecuatoriana. Revista Ciencia y Trabajo. | AÑO 16 | NÚMERO 51 | SEPTIEMBRE / DICIEMBRE 2014.

Aliaga, P., Villarroel, J., \& Cossio, N. (2016). La charla motivacional: Una estrategia para abordar el desconocimiento de factores de riesgo ergonómico en un supermercado chileno. . Ciencia \& Trabajo.

Almirall, P., \& Marroquín, E. (2016). Ergonomía cognitiva. Resultados de un taller de capacitación. Revista Cubana de Salud y Trabajo.

Angulo Hurtado, A., \& Ramírez Quintana, A. (2016.). Relación entre la calidad de vida en salud y la carga física en cuidadores de personas con enfermedad de Alzheimer. . Revista Colombiana de Salud Ocupacional.

Bettiol, M. (2016). Riesgos del trabajo: un giro se produjo en la Argentina. En Superintendencia de Riesgos del Trabajo. Veinte años del Sistema de Riesgos del Trabajo.

Bravo Carrasco, V., \& Espinoza Bustos, R. (2016). Factores de Riesgo Ergonómico en Personal de Atención Hospitalaria en Chile. Ciencia \& trabajo.

Caicedo, A., Manzano, J., Gómez, D., \& Gómez, L. (2015). Factores de Riesgos, Evaluación, Control y Prevención en el levantamiento y Transporte Manual de Cargas. Revista Colombiana de Salud Ocupacional.

Callizo, M. D. (2015). Prevención de riesgos laborales en Paraguay: Principales consideraciones. Revista de la Facultad de Derecho, (39), 2-2.

Cavas Martínez, F. (2016). Aspectos jurídicos de la enfermedad profesional: estado de la cuestión y propuestas de reforma. . Medicina y Seguridad del Trabajo, 62., 78-86.

Chaves, M., Martínez, D., \& López, A. (2014). Evaluación de la Carga Física Postural y su Relación con los Trastornos Musculoesquelético. Revista Colombiana de Salud Ocupacional.

Constitución de la República del Ecuador 2008. (2008). Constitución de la República del Ecuador. Artículo 326, numeral cinco. Montecristi: Ruiz.

Escudero, E., Aprili, L., Muñoz, V., De La Cruz, M., \& Moscoso, M. (2016). Prevalencia de síndrome del túnel carpiano de origen laboral en odontólogos de la ciudad de Sucre. . Revista Ciencia, Tecnología e Innovación - Bolivia.

Galvis, F., Pérez, J., Ramírez, Y., Batancur, C., \& Gómez, L. (2015). Carga Física en Trabajadores del Área de Acabados en Industria Metalmecánica. . Revista Colombiana de Salud Ocupacional. .

Gómez-Ceballos, D. A. (2016). Accidentes de trabajo y enfermedades laborales en los sistemas de compensación laboral. Revista Brasileira De Medicina Do Trabalho. 
Guizado Ramos, M., \& Zamora Cordova, K. (2014). Riesgos ergonómicos relacionados a la lumbalgia ocupacional en enfermeras que laboran en Centro Quirúrgico del Hospital Daniel Alcides Carrión.

Hodelín, Y., De los Reyes García, Z., Hurtado Cumbá, G., \& Batista Salmon, M. (2016). Riesgos sobre tiempo prolongado frente a un ordenador. Revista de Información Cientifica - Universidad de Ciencias Médicas de Guantánamo.

Kamei, T., Takahashi, K., Omori, J., Arimori, N., \& Hishinuma, M. (2016). Por una enfermería de práctica avanzada y una alianza para la atención centrada en las personas, para la Cobertura Universal y Acceso. Revista Latinoamericana de Enfermagen.

Montalvo, A., Cortés, Y., \& Rojas, M. (2015). Riesgo Ergonómico Asociado a Sintomatología Musculoesquelética en personal de enfermería. Hacia la promoción de la Salud, vol.20 núm 2.

Morán Ramírez, C. (2016). Riesgos laborales del profesional de enfermería en los quirófanos del Hospital Sergio e. Bernales-Collique. Revista Peruana de Obstetricia y Enfermería.

Moreno, M. (2016). Ergonomía En La Práctica Odontológica. Revista Revencyt.

Oñate, L. D., Chica, M. P., Jaraba, D. C., \& Jaraba, M. A. (2015). Determinación de los factores de riesgo ergonómico a nivel de miembro superior en los trabajadores del área administrativa de la Fundación Médico Preventiva en la ciudad de Valledupar, Cesar. Revista Agunkuya, 2(1), 22-31.

Ordoñez, C., Gómez, E., \& Calvo, A. (2016). Morbilidad sentida osteomuscular en trabajadores administrativos de una empresa metalmecánica. Revista Colombiana de Salud Ocupacional.

Pereira Gomes, L., Da Silva Garzedin, D., \& Dominguez Ferraz, D. (2016). Impacto del lumbago en la calidad de vida de los trabajadores: una búsqueda sistemática. Revista Salud de los Trabajadores.

Pinto, R. (2015). Programa de Ergonomía Participativa para la prevención de Trastornos Musculoesquelético. Aplicación en una empresa del Sector Industrial. . Revista Ciencia y Trabajo.

Profesores de la Universidad de Santiago de Chile. Malchaire Jacques. (2016). Estrategia Sobane Máquinas y Herramientas. Revista Salud de los Trabajadores.

Rincones Ortiz, A., \& Castro Calderón, E. (2016). Prevención de desórdenes musculoesquelético de origen laboral en Colombia. Revista de Ciencias de la Salud.Carpenter (1961). En D. Gonzales, Ergonomía y Psicosociología (pág. 37). . FC Editorial.

Sbriller, L. (2016). Consideraciones sobre la inclusión de Terapia Ocupacional en riesgos del trabajo. Revista Argentina de Terapia Ocupacional. 2. 
Serra Valdés, M. (2016). Las enfermedades crónicas no transmisibles: una mirada actual ante el reto. . Revista Finlay.

Soriano Tarín, G., Rodríguez-Caro, M., Pascual Sagastagoiti, I., \& Del Campo Balsa, M. (2016). Estudio sobre el envejecimiento activo saludable y su relación con las condiciones de trabajo en el sector sanitario. Revista de la Asociación Española de Especialistas en Medicina del Trabajo.

Souza de Souza, R., Cortez Antunes, E., Gomes, D. C., T., \& Santana Ferreira, R. (2016). Enfermedades profesionales de los trabajadores de limpieza en los hospitales: propuesta educativa para minimizar la exposición. Digitum: Revista Universitaria.

Zamora, Y., Montesdeoca, M., Negrin, E., \& Párraga, J. (2016). Riesgos Laborales trabajadores centro de acopio Almidón de Yuca Sitio Tarugo. . Revista ECA Sinergia.

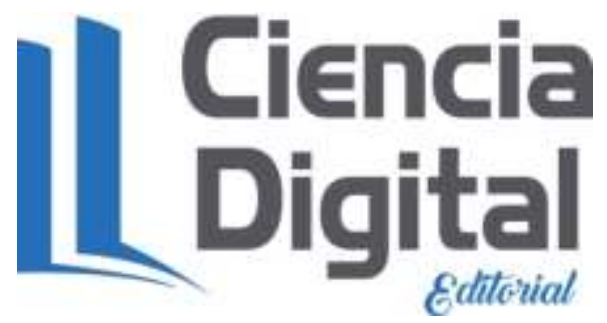

Para citar el artículo indexado. 
Vargas M., Ubilluz M., Vega G., Fiallos P., \& Núñez C. (2018). Los riesgos ergonómicos en los trabajadores del hospital básico Baños. Revista electrónica Ciencia Digital 2(1), 127146. Recuperado desde:

http://www.cienciadigital.org/revistascienciadigital/index.php/CienciaDigital/article/view/9 19

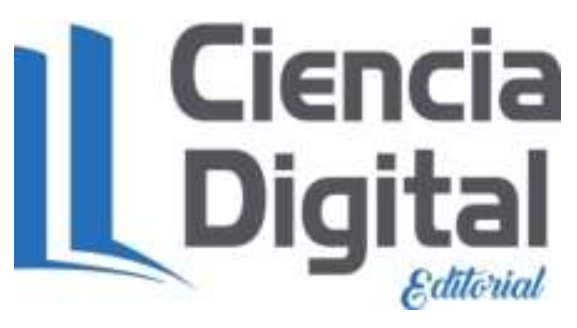

El artículo que se publica es de exclusiva responsabilidad de los autores y no necesariamente reflejan el pensamiento de la Revista Ciencia Digital.

El articulo queda en propiedad de la revista y, por tanto, su publicación parcial y/o total en otro medio tiene que ser autorizado por el director de la Revista Ciencia Digital.
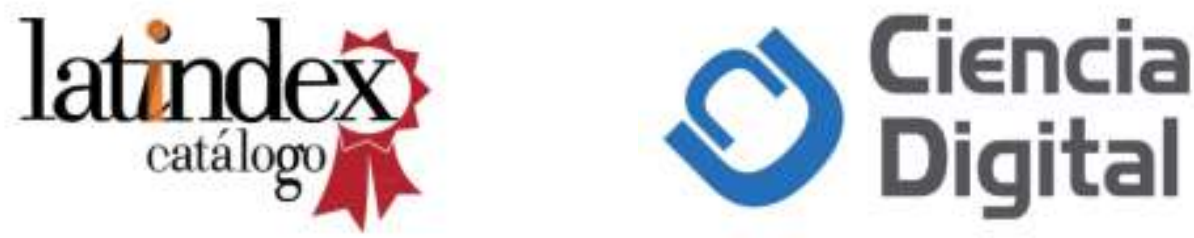